Asian Journal of Information Technology 18 (3): 89-98, 2019

ISSN: $1682-3915$

(C) Medwell Journals, 2019

\title{
Statistical Data Analysis Procedures in Mass Communication Research
}

\author{
${ }^{1}$ Joseph Oluchukwu Wogu, ${ }^{2}$ Christiana Ogeri Chukwu, ${ }^{2}$ Chukwuemeka Ononuju Nwankiti and \\ ${ }^{1}$ Chinyere Christiana Ugwulor-Onyinyechi \\ ${ }^{1}$ Department of Mass Communication, University of Nigeria, Nsukka, Nigeria \\ ${ }^{2}$ Department of Mass Communication, Alex Ekwueme Federal University, \\ Ndufu-Alike Ikwo, Nigeria
}

\begin{abstract}
The purpose of this study is to examine statistical data analysis in mass communication research. There are three major methods of analysing data in mass communication research, namely: content analysis, discourse analysis and qualitative/computational analysis. None of these methods is unique or exclusive to mass communication as they are interdisciplinary. However, research activities in the discipline of mass communication in the 21 st century are bereft of discourse analysis. Guided by the principles of object-oriented database theory, we argued that the procedures for data analysis in mass communication research are not unique to analysis procedures in other academic disciplines. Considering the observations made in the course of discussions and opinions reached, this study offers some recommendations as proposed solutions.
\end{abstract}

Key words: Data analysis, mass communication, content analysis, discourse analysis, computational analysis, object-oriented database

\section{INTRODUCTION}

Guided by the principles of object-oriented database theory, we explored why and whether the procedures for data analyses in mass communication are not inferior or unique to analysis procedures in other academic disciplines. The principles and methods of data analysis in mass communication research are interdisciplinary in nature. Mass communication research shares in the principles and rules of the various methods of data analysis being used in other academic disciplines/fields and their defects. Data, as used in this, study is a symbolic representation of and raw material people use to investigate social processes, policies and programmes. It is therefore, a by-product of social process, policies and programmes whether public or private. Thus, data becomes the appropriate instrument for the evaluation of the social process, policies and programmes. It is a distinct piece of information that exists which exists in a variety of forms such as numbers or text on pieces of paper, bits and bytes stored in electronic memory or as facts stored in a person's mind. Characteristically, data exist either in qualitative or quantitative form.

Analysing data, therefore, refers to action taken on data which provides and/or assigns meanings to the data and enables the analyser to extract and communicate information about the social process, policies and programmes from where the data was generated. Therefore, in the opinion by Marshall and Rossman (1999), data analysis refers to "the process of bringing order, structure and meaning to the mass of collected data". It is a process that searches for and generates a general statement concerning the meaning and implications of categories of data and requires some forms of logic wherein deductive and inductive reasoning are applied in order to generate interpretations and predictions (Seward et al., 2012). Therefore, we are of the opinion that data analysis is a tool and also a mechanism for communication in academic and social processes.

There are many approaches to and methods of data analysis in research but are adopted differentially by different academic disciplines for specific inquiry and/or as a disciplinary culture. However, the manner these approaches and methods are adopted, personalised and presented for data analysis by individuals, different academic disciplines, practitioners and professionals helps to determine the quantity and direction of information flow in society. The character of this information flow on its part enables stakeholders to determine the behaviour of social processes and the society's ability to control them (Welsch, 1992).

Therefore, the primary purpose of data analysis is to obtain usable, applicable and useful information from social processes in order to comprehend and control social outcomes. Such analysis, irrespective of whether the data is qualitative or quantitative tends to describe and summarise data, identify relationships between variables that are associated with the data, draw comparison among the associated variables, identify the differences among variables, their relationships and the causative factors that

Corresponding Author: Chinyere Christiana Ugwulor-Onyinyechi, Department of Mass Communication, University of Nigeria, Nsukka, Nigeria 
structured the relationships, the consequences of their relationships and forecast future outcomes. In my opinion, these concepts, processes and outcomes that characterise data and its analysis in different academic disciplines are similar to research experiences or are replicated in mass communication research also. Thus, they are inter-disciplinary and not the exclusive reserve of any discipline.

\section{MATERIALS AND METHODS}

This study is a perspective on statistical data analysis in mass communication research. The study is guided by the principles of object-oriented database model or theory as propounded or espoused by its protagonists such as (Millen and Lunt, 1992). The focus of object-oriented data theory is object-oriented programming whose basic element is the object. According to this theory, an object works as a run time entity with a unique identity, states and behaviours. Therefore, objects differ and are classifiable with appropriate and different definitions that align with or explain their various identities, states and behaviours. According to Millen and Lunt (1992), classes are arranged in a hierarchal form as logical containers of data and methods which are used to perform operation on the data items. In this hierarchy is the root cause or variable that beget others known in a database as a parent class and other classes that inherit or make use of the properties of parent class known as child class. In general social science, education and arts research language they are known as independent and dependent variables. According to the principles of object-oriented data theory, these properties that are inherited from the parent class are amenable to modifications during the process by intervening variables to produce new results or products that define the relationship. It also provides reusability of data and methods in the mean of inheritance. The applicability of the object-oriented database theory to this study lies in its provision for and identification of classes and/or categories of data that differ from each other but interact to generate results. It enables the data analyst in mass communication research to look for and identify the parent class (independent variable) and the child class (dependent variable), explore their properties and identify areas and character or manner of inheritances exhibited by the dependent variable and define their relationship with the independent variable (child class). The theory enables the study to form its opinions with regards to data analysis procedures in mass communication research.

\section{RESULTS AND DISCUSSION}

Data analysis in mass communication research is of two categories or types, namely: qualitative or content or non-science or humanism analysis and quantitative analysis or mathematical computations. This view is supported by Moy and Murphy (2016). As used here and in the opinion by Alshenqeeti (2014), qualitative data analysis refers to "the process of making sense from research participant's views and opinions of situations, corresponding patterns, themes, categories and regular similarities". Characteristically, this form of analysis tends to be an on-going and iterative process where the information collected is transformed by means of analytic procedures to explain and predict issues. It seeks to establish a converged and general statement about subsisting relationships between categories of variables.

In the opinion by Creswell (2013), qualitative data analysis focuses on and investigates original researchers or pieces of raw information their intentions, the patterns and/or themes associated with the raw information, sift pertinent issues from trivia ones, establishes the regularity of their use or appearance, determines their similarities and differences and the complexity of the phenomenon under study. It is simply a codification of common sense and not the complexities of figures or statistical data.

Nevertheless, there is a conflict of opinion among scholars on the specific guideline or best and precise method for analysing qualitative message or text or data. The implication of this conflict of opinion or lack of consensus of opinion is that there are many approaches or methods that can be applied in analysing qualitative data. This prompted McKee (2001) to opine that "the specificity of any methodology must be investigated to reveal the limits to the kinds of knowledge it can produce and yet our own central methodology is woefully under-investigated and still largely intuitive". Nonetheless, mass communication researchers can and do adopts text analysis, narrative analysis, rhetorical analysis, discourse analysis, content analysis, interpretative analysis and semiotic analysis as well as some of the techniques used in literary studies such as critical analysis. However, we strongly contend that these forms of qualitative data analysis are not the prerogative of mass communication researchers alone. They are adopted or utilised by other academic disciplines where the use of content analysis in research procedures has been cultural.

Figure 1 shows the principles for analyzing data in mass communication involve the collecting of like terms, noting of the frequency of the recurrent event, observing the prevailing patterns and trends in data, establishing the differences, splitting the inherent variables, identifying intervening variables, categorizing issues properly and identification of relationship and factors. Irrespective of any of method or approach any researcher may choose to adopt for successful data analysis, we support (Miles and Huberman, 1994) opinion which holds that the researcher must be guided by or adhere to the following principles: 


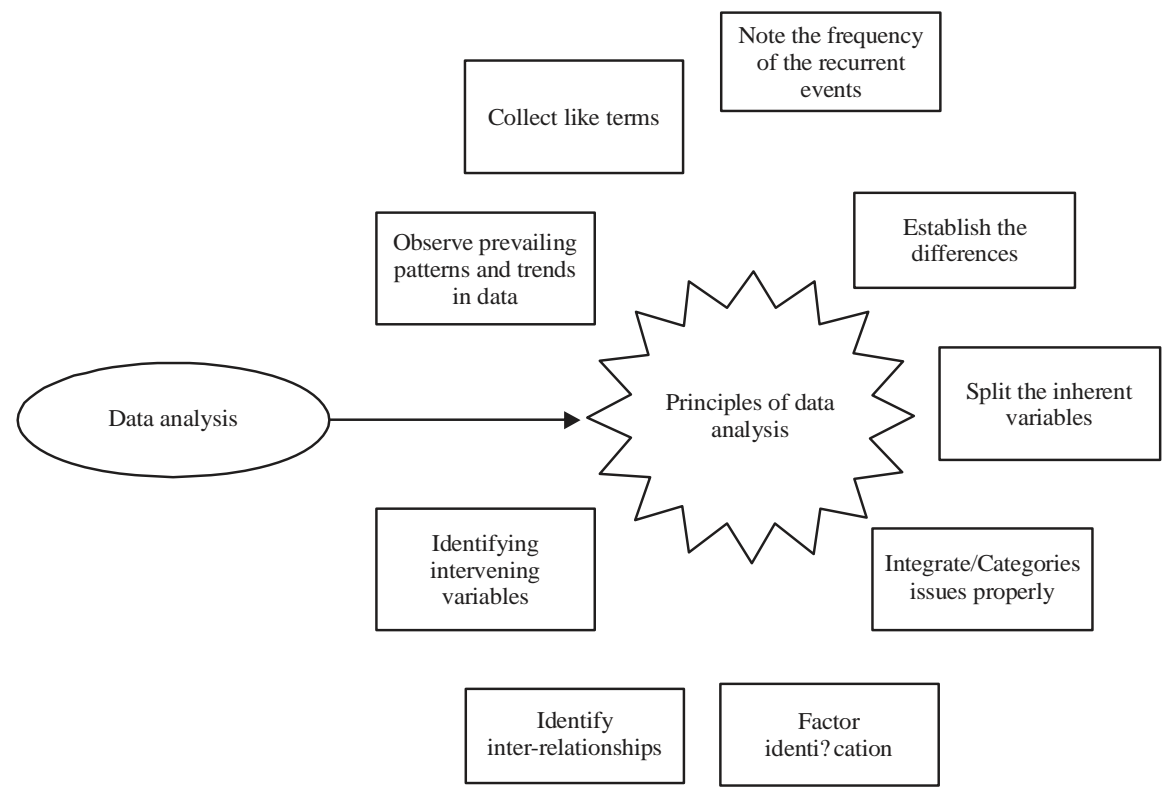

Fig. 1: Data analysis procedures in mass communication research

- Note the prevailing patterns and trends in the raw information being studied to determine, if they make sense

- Group the events, places and people found in the raw information together, if they appear to have similar patterns or characteristics

- Count the frequency of the occurrence of the recurrent events in the raw information

- Establish observed similarities and differences between and within the raw data sets through comparison

- Split the inherent variables in the raw information with a view to finding more coherent descriptions and explanations of their relationships

- Integrate or subsume particular issue found in the relationships into the general culture or template, that is link specific data issues to general concepts, theories and categories in the discipline

- Identify the factors or variables that are responsible for the processes under investigation

- Identify the inter-relationships between different parts of the raw data variables and the consequences of such relations

- Identifying the intervening variables in the relationships

- Build a logical chain of evidence by developing logical trends in the relationships

- Declare the prevailing theoretical coherence in the relationship which serves as a generalisation that may be tested by others irrespective of location

On the other hand, quantitative method of data analysis refers to the form of analysis that investigates the relationships that exist among different sets of data. This type of analysis uses scientific tools such as a spreadsheet, statistical packages, MAXQDA, ATLAS, Dedoose, QDA MINER and NVivo among others. The quantitative method of data analysis is a systematic method of investigations whose process involves only numerical data collection and the transformation of the raw information collected concerning a situation or event into numerical data in search of answers to questions like 'what', 'why', 'how' and 'how many'.

The main features and focus of quantitative method of data analysis are surveys, statistics and measurement, pre-existing data and questionnaires. The analysis seeks to find out the frequency distribution contained in the data set, the percentage distribution of the frequency, the central tendency and deviations inherent in the data set. This method of analysis involves measuring and counting attributes or variables in search of evidence to either support or contradict a pre-existing idea or hypothesis. A hypothesis refers to the tentative answer given to a question before verification of facts begins.

In support of the opinion by Creswell (2013), we state that qualitative data analysis focuses on data collected from a natural setting where researchers are the key instruments of data collection and analysis. In this method of analysis, researchers apply both inductive and deductive reasoning to arrive at their inferences. The primary intention of researchers during the analysis is to construct meaning out of the statistical data and with the aid of emergent research designs, explore and develop the ideas generated for possible generalisation. This method is characteristically flexible, iterative and factors-in the complex relationship of the researcher during the fieldwork experiences. 
We uphold the view that the processes of quantitative data analysis are custom-built and rarely 'off-the-shelf' . This is because most of its tools of analysis are in software form while other instruments are various formulas developed for such purpose. Any form of deviation from the principles and methods of this software and formulas is an aberration and error. Therefore, in the opinion of scholars like Charmaz(2006), quantitative data analysis requires researchers to:

- Read transcripts and data collections methods and products several times and holistically before trying to break it down into parts for analysis

- Develop between 5-6 coding categories and then revisited them for expansion which should not exceed 25-30 categories. The codes are then incorporated into thematic 'families' which are dependent upon the methodological approach

- Use the exact words of participants as a particular code i.e.,vivo codes

- Create and organize files for data

- Read through text, make margin notes, form initial codes

- Use of line-by-line or segment-by-segment data

- Describe open coding categories (GT)

- Describe the social setting, actors, events as coded

- Select one open coding category for analysis

- Analyse data for themes and patterned regularities

- Interpret the data by selecting coding to interrelate categories to form a 'story' or hypothesis (GT)

- Discover how the culture works and create meaning out of the themes

- Present data through a narrative presentation which is supplemented by figures/visual aids

In our own opinion, the presentation of the narrative which may differ from one researcher to the other is a function of individual ability and interpretation which is structured by the cultural, social, gender, class and personal politics that brought such individual into the research. It, therefore, holds that all data analysis narratives are executed within a framework that is always personalised. Simply put, researchers shape research analysis and the associated report that emerges from it. This is not alien to research experiences in the field of mass communication. Academics or research in mass communication research apply both qualitative and quantitative methods separately and collectively and are not immune to the researcher's shaping of the analysis process and reports too (Wimmer and Dominick, 1994). Both qualitative and quantitative methods of data analysis apply also in the academic field/discipline of sciences, arts and humanities.

Research traditions in mass communication as well as social sciences and arts, show that qualitative or content or non-science or humanism data analysis is composed of two separate methods (Anderson, 1987). These methods are holistic analysis or interpretive studies and value-based judgments or criticism.

According to the opinion by Moerman (1992), the primary focus of the interpretive approach is "to find out how the events he observes and experiences in the alien world make sense to the aliens how their way of life coheres and has meaning and value for the people who live it". The subsisting primary objective of the approach is for the researcher to understand human behaviour from the actor's own frame of mind and behaviour. The approach, therefore, jettisons the study of a set of isolated variables and explores dynamics across contexts and a holistic mixture of attributes.

On the part of value-based judgments or criticism, researchers focus on analysing values and judging or criticizing such values. Accordingly Leah et al. (1991), noted that "the primary purposes of criticism are understanding, explanation and appreciation (of) socio-cultural, symbolic form". This approach tends to investigate the variable or factor that creates hegemony in the phenomenon of study such as people, events, phenomena, artefacts, etc. and whether the values of the established hegemony can be altered, how and when? In this approach judgments which are products of the researcher's ideological leaning are taken as data. Researcher's critical investigations which reveal judgments regarding the centre of hegemony can be contested by other researchers within and outside the same discipline.

There are three alternative methods of applying qualitative content analysis to mass communication research. These alternatives are differentiated by the degree of inductive reasoning required or applied. This view collaborates (Hsieh and Shannon, 2005) opinion. First, there is the conventional qualitative content analysis which is used when inductive analysis flows directly from the raw data. This enhances grounded theory development. Second, there is the directed content analysis which is used when the inductive analysis flows directly from a theory or relevant research findings rather than raw data. This alternative is used to validate or extend a conceptual framework or theory. Third, there is the summative content analysis which is used when analysis or counting of concepts or words are involved. This alternative explores the usage of the words inductively.

However, data analysis in mass communication research like its counterparts in other disciplines is structured by three different scales of analysis, namely: nominal, ordinal and numerical scales. A nominal scale concerns data that are classifiable into non-numerical or named categories which are generated and/or classified in an arbitrary manner. The ordinal scale is concerned with 
data that is classifiable into non-numerical or named categories but with prevailing inherent order existing among the response categories. This exists in questions that call for ratings of quality (such as very good, good, fair, poor, very poor) and agreement (such as strongly agree, agree, disagree and strongly disagree). The numerical is concerned with numbers that represent the response categories which is normally ranked or categorised from zero upwards. The categorisation exhibits clear quantifiable difference within categories and between consecutive categories such as $5,4,3,2,1$ and 0 .

Similarly, content analysis which is fundamental to communication research is a celebrated and one of the primary methods of analysis in all the disciplines of social sciences, arts and humanities (Lacy et al., 2015). Simply put, content analysis is an interdisciplinary method 'for the objective, replicable and quantitative' description of text's. Concurring to this opinion, Lombard et al., noted, "given that content analysis is fundamental to communication research (and thus, theory) it would be logical to expect researchers in communication to be among the most, if not the most, proficient and rigorous in their use of this method".

In mass communication research as well as in other disciplines, content analysis has four primary functions in research. These functions are as follow: "to produce increases in the degree of confirmation of hypothesis already generally, presumed to be valid and definitive disconfirmation of hypothesis already generally presumed to be invalid. To correct "optical illusions" which may be shared by most specialists. To settle disagreements among specialists as the truth value of certain propositions. To permit the formulation and the testing of hypothesis" (Franzosi, 2008).

Most of the mass communication researchers, like their counterparts in other disciplines and fields, systematically evaluate the symbolic contents of all forms of recorded issues concerning their phenomenon or subject of inquiry. The research by Pasadeos et al. (1995) validate this opinion. It is through evaluation, deductive and inductive reasoning that researchers extract meaning and draw inferences from these recorded issues. These recorded issues include among others: words, images, roles, audio, video etc. Details of the process of and procedures for evaluating recorded issues involve the identification, coding, recording of relatively objective or at least inter-subjective characteristics of the manifest contents of the subject of inquiry and their interpretations. These processes have earlier been attested to by Schilling (2006). The process begins with identification and data preparation. Content analysis is a veritable tool for analysing various types of data and the method of analysis depends on the type of data. Therefore, the type of data must be identified first and then transformed into written text (i.e., data preparation) before analysis can start. In a situation where the data exists as texts derived from other sources, the researcher must justify his choice of the content and source. This opinion collaborates the views by Schilling (2006). For example, in analysing an interview transcripts which seeks to reveal interviewee's behaviours and thoughts, the researcher must determine whether to use all questions posed by the interviewer or select from among them whether to include all the issues raised by the respondent or select from among them whether the responses should be transcribed literally or only in a summary and whether to include or consider side variables such as sounds, pauses, external voices and other audible behaviors that manifested during the interview. In all these, the researcher must justify his decision or selection for scientific reasons.

After data preparation, the researcher defines the unit of analysis to be employed, that is the basic unit of text to be classified in the process of content analysis. De Wever et al. (2006) are of the view that the unit of analysis specification enables effective coding of data. In qualitative content analysis, individual themes are always used as the unit of analysis instead of word, sentence or paragraph. In this method, Minichiello et al. (1990) are of the opinion that the researcher should primarily consider and look for the expressions of an idea contained therein. The researcher can assign a code to any size of text chunk, so, long as it represents a single theme or issue of relevance to the research question. After coding the entire data set, the consistency and reliability of the coding system should be checked and rechecked. It is not safe to assume that the coding is consistent and reliable because human coders are subject to fatigue and naturally prone to error.

The next step involves the development of categories and a coding scheme that serves as units of measure and evaluation. The categories and coding scheme can be derived from the data for the study, previous but related studies and existing theories in the field. They should be defined to ensure internal homogeneity and external heterogeneity. This opinion collaborates Lincoln and Guba (1985) view. To achieve a fairly standardized process in the process of analysis, the schemes developed must be tested to validate and reliability. The best approach here is to code a sample of the study data and the coding consistency checked by assessing its inter-coder agreement. This opinion is supported by Schilling (2006) who noted that if the result of the assessment is low, the coding rules must be revised.

In the coding of the observed and recorded manifest contents of the phenomenon of inquiry, the degree of percentage agreement popularly known as simple agreement or percentage of agreement is adopted as a mechanism for drawing an inference or taking a decision. This method is also pervasive in other disciplines and 
fields. In the process, the researcher pairs coders to find out their degree of agreement or disagreement (Kolbe and Burnett, 1991). This index is simple, intuitive and easy to calculate. In the opinion by Miles and Huberman (1994), the sequence of this process is as follows:

- "Giving codes to the initial set of materials obtained from observation, interviews and documentary analysis

- Adding comments and reflections (commonly referred to as memos)

- Going through the materials trying to identify similar phrases, patterns, themes, relationships, sequences and differences between sub-groups

- Taking identified patterns and themes out of the field to help focus the next wave of data collection

- Gradually, elaborating a small set of generalisations that cover the consistency one discerned in the data

- Linking the generalisations to a formalized body of knowledge in the form of constructs (theories)"

The next phase is the interpretation of coded data. The focus of this step is to develop and assign meaning to the coded themes and their properties. This will lead to inferences and reconstructions of meanings as derived from the coded data. In my view, the method here is to explore the properties and dimensions of the categories, identify the relationships between them and explicate the patterns of the relationships. The success of this stage is subject to the reasoning ability of the researcher. Thus, I uphold the opinions by Potter and Levine-Donnerstein (1999) among others which held that the interpretation of coded data is primarily organised and empowered by the mental schema of the researcher.

The interpretation process is typically descriptive in nature, relying on simple descriptive statistics, the researcher's skill/analytical ability and pre-existing tradition/findings in the field (Clark and Illman, 2003). Therefore, data interpretation relies heavily on the researcher's study of previous or existing and appropriate materials, previous knowledge and the interpretative ability for a reliable conclusion to be reached. It is pertinent to note that content analysis does not produce counts and statistical significance but explicates patterns, themes and categories that are essential for managing social realities.

The final stage in content analysis is the presentation of the research findings. At this stage, the researcher presents his conclusions or inferences and in many cases, uses typical quotations to justify the conclusions reached (Schilling, 2006).

The pitfalls or experienced weaknesses of content analysis led to the emergence of discourses analysis in mass communication research in the early 1970 s. This form of data analysis attends to or focuses on the 'surface' properties of presentation, layout, graphical display in printed discourse or intonation, para-verbal and nonverbal features in spoken media discourse, syntactic structures, lexical style or rhetorical devices, underlying meanings and connotation inherent in the phenomenon or subject of study. Nevertheless, the object of study in this case is the media, news, advertising, film, TV-programs such as talk shows, etc. (Van Dijk, 1985).

However, this method of analysis i.e., discourse analysis is not restricted to mass communication alone. The method is combined with content analysis and applied by researchers in arts and social sciences disciplines such as political science, sociology among others in pursuit of decision or inference during research and analysis. Therefore, discourse analysis is not exclusive for mass communication researchers.

Similar to these inter-disciplinary methods is the use of quantitative methods of analysis in mass communication research. Quantitative analysis or mathematical computational analysis typically analyse data generated in pursuit of specific and clearly defined questions that examine the relationship between two events or occurrences. The second event is always a consequence of the first event. It tests the causality or link between the two events or issues using data gathered through surveys and carefully developed and structured questionnaires.

Unlike qualitative methods, this form of analysis deals squarely with figures and not words. Thus, quantitative data analysis uses formulas; Excel spread-sheet, Microsoft access database and/or statistical software in pursuit of central tendencies, range and deviations in a data set. Due to technological advancement, a range of specialist software for undertaking quantitative data analysis is now available and accessible. Such software includes the popular Microsoft Excel (that can be used to run basic descriptive statistics as well as a range of more complex statistical analyses) and the Statistical Package for Social Sciences (SPSS). The software contains a range of tools for descriptive statistics which can be used to describe, analyse and/or summarise data. These tools include:

- Frequency: the 'count' or number of responses in each category

- Percentage (\%): the proportion of people who give a particular response or set of responses

- Mean: the arithmetic average that uses standard deviation to illustrate variance

- Standard deviation: the average spread of scores around the mean

- Median: the middle value

- Mode: the most common response to a question and

- Range: the difference between the highest and lowest response value

A range of other statistical tests such as Chi-square, cross-tabulations and pairwise comparison among others are also available to help mass communication researchers 
establish the significance of any differences between results. However, their applicability is not exclusive to mass communication discipline alone.

Consequently, the methods and processes of quantitative data analysis or mathematical calculations in pursuit of central tendencies, range and deviations in mass communication adhere strictly to the scientific methods being used in other academic fields/disciplines. Popular opinion in the literature holds that this process of analysing data involves elements such as objectivity/ inter-subjectivity and a priori design. This form of analysis is "used widely in mass communication as a way to count manifest textual elements, an aspect of this method that is often criticized for missing syntactical and semantic information embedded in the text" (Weber, 1990).

The major goal of scientific investigation and analysis in mass communication like in other fields/disciplines is to explain data through processes or formulas that eschew or minimise researcher's biases. These processes provide for consistency accuracy and inter-subjectivity (Babbie, 1989; Neuendorf, 1989). To achieve this and curtain the inherent weaknesses of content analyses towards objectivity that tend to manifest in researcher's continual construction new list of issues in the process of study, a prior research design which contains "all decisions on variables, their measurement and coding rules must be made before the observation begins" (Neuendorf, 1989). This application of this method which is called the deductive method of data analysis is pervasive in all disciplines/fields of study, mass communication inclusive. The principles governing quantitative data analysis in mass communication research are the same in all disciplines/fields of academic research. These principles are:

The organisation of data: All forms/questionnaires used in gathering responses are assembled and checked for completeness and accuracy of responses and mistakes are identified and removed while each form/questionnaire is assigned a unique identifier.

Data collation: Responses or respondents accent to each category of response options are gathered and entered manually or into a computer to enable frequency count, percentage, central tendency and deviation calculations, ranking and cross-tabulation.

Interpretation of information: Meaning is attached to the data set and numerical results generated from the calculations. Figures do not speak, therefore, meanings are attached to them by the researcher. It must be noted that different meanings can be attached to the same figure depending on disciplinary traditions, orientation and ability of the researcher. For instance, $75 \%$ can be given a positive or negative interpretation or meaning depending on the research context and question. Such interpretation tries to address impact or influence, variable role or contribution, lesson or discovery etc.

Identification of limitation: For a possible explanation of any deficiency in the analysis, limitations or challenges encountered in the process must be mentioned or stated. This will justify any possible deviation or difference from similar analysis or researcher any other time and place.

Conclusion and recommendation: A precise statement on the discovery or finding of the analysis should be made. In this, the researcher states any area or issue relating to the project that was either not understand or resolve. On this frame, an appropriate recommendation is offered for further research and analysis.

We hold the view that the ascendency or contemporary dominant preference for quantitative data analysis in mass communication research as evident in most of the current research publications/reports and among academics in Nigeria is not the norm. Many scholars substantially argued in favour of this opinion. Conceding to this no superiority argument, Amadi (2014) is of the opinion that quantitative data analysis equally possesses certain weaknesses. According to his opinion, quantitative data analysis does not enhance "knowledge and social progress as its outcomes are intended to satisfy the corporate interest of sponsors of social research". Therefore, some scholar's insistence on quantitative analysis over qualitative analysis in many mass communication departments in Nigeria as the most objective form of analysis does not hold. We are of the opinion that their Americanised influence and preference for quantitative analysis, though good for research analysis that pursues the desired result using survey and deals with effects or influence cannot transmogrify into superiority and/or under-write the objectivity and positive contributions of qualitative analysis.

Although, we concede to the opinion by Kohlbacher (2006) which holds that "qualitative research methods have often faced acceptance problems and academic and disciplinary resistances which are partly due to the politics embedded in this field of discourse" it is not absurd to adopt it as a method of research and analysis. Both forms of analysis, that is quantitative and qualitative are objective, acceptable and reliable in communication research and analysis as in other disciplines (Ellingson, 2012). Many scholars such as Bryman (2004), share the opinion that both methods are complementary rather than rivalries. This admission has led to the adoption or application of mixed method approaches and the use of triangulation in many types of research and analyses. From the second half of the 20th century into the $21^{\text {st }}$ 
century, there is manifest advances and results in the application of qualitative methods of analysis in research not only in the field of social research.

The qualitative methods use words such as concepts, terms, symbols, etc., to construct a framework for communicating the essence of what the data reveal. On the other hand, quantitative methods employ procedures, techniques and numbers to construct its own framework for communicating the essence of what the data reveal (Sesay, 2011). However, both methods share similar characteristics in several ways. For instance, both of them use reasoning to reach conclusions that are based on evidence reveal their study design in some ways employ comparison (i.e., identification of patterns that are similar or different) as a central process of data processing; strive to avoid errors, false and misleading conclusions; apply deductive and inductive logic in the course of data processing in every research and share the common purpose or goal of conducting any study which is to produce findings and/or answers to existing questions (Bushko, 2009).

Therefore, any of the methods can be objectively applied depending on the subject of inquiry and nature of data to be analysed. This opinion is in line with the growing consensus of opinion among social scientists (Amadi, 2014; Denzin and Lincoln, 2012) which holds that the nature or character of the subject of study determines the method that should be adopted for it.

The above opinion suggests, therefore, that both qualitative and quantitative methods are not similar and interchangeable. In the opinions of certain scholars like Kreuger and Neuman (2006), there exist categorised and verifiable differences between the two. Some of these differences include.

In qualitative data analysis, there are a wide variety of approaches to research processes compared to quantitative method wherein researchers choose from among a few pre-existing standard sets of data analysis techniques or formula.

In the qualitative method, the product of data analysis structures and guides subsequent data collection and analysis while in the quantitative method of analysis, the analysis itself does not begin until all data have been collected and condensed into numbers and its product does not structure or guide subsequent data collection and analysis.

In the course of qualitative data analysis, new concepts and theories can or are created by researchers, while in the quantitative method, analysis pursues the validation or invalidation of proposed hypothesis with variable constructs and data analysis in the qualitative method is imprecise, diffuse and context-based because it deals with words while in quantitative method, the language of analysis is the figure, statistical relationship and is therefore, more precise and replicable.
It is our objective view that the various approaches to data analysis being used in mass communication research adhere to the procedural rules evidenced in other disciplines of study. These approaches or methods are generally, structured by the purpose of the study (every research has a specific objective), theories (categorised into social scientific theories, interpretive theories and critical theories), hypothesis (exploration of measurable tendencies among individual attributes or variables being studied), methodology (adopting specific pattern or method from among multiple patterns available to researchers), sampling selecting the set of data for analysis from a larger population), interpretation, conclusion and generalization (describing the conclusion reached on the data across a population from the part of population that was studied). This view collaborates the opinions expressed (Wimmer and Dominick, 1994).

\section{CONCLUSION}

Considering the observations made in the course of discussions above and opinions reached, this study offers the following recommendations. First, the debates and discussions on the superiority of the quantitative method of data analysis over qualitative method have been objectively flawed among scholars, theoreticians and researchers. Therefore, both methods are objective and should be accepted whenever and wherever used provided their respective procedures and rules were adhered to. None should be rejected or preferred against the other so long as the circumstantial adoption is justified and objective. Scholars should eschew the dichotomy and focus on modernising each method to address contemporary challenges. To resolve the conflict of opinion subsisting among scholars concerning the specific guideline and precise method for analysing qualitative data, we are of the opinion that all the methods being used by scholars irrespective of region, culture, ideology or level of development should be endorsed and harmonised for general applicability. To achieve this, international workshop/conference should be organised by research experts/professionals with a goal to articulate specific template or guideline for each of the prevailing methods of qualitative data analysis just like the development of formulas for quantitative data analysis.

\section{REFERENCES}

Alshenqeeti, H., 2014. Interviewing as a data collection method: A critical review. English Ling. Res., 3: $39-45$.

Amadi, F.A., 2014. Trends in mass communication research in Nigeria: A guerrilla warfare dissertation. Asia Pac. J. Multi. Res., 2: 78-86. 
Anderson, J.A., 1987. Communication Research: Issues and Methods. McGraw-Hill, New York, USA., ISBN:9780070016514, Pages: 423.

Babbie, E., 1989. The Practice of Social Research. 5th Edn., Wadsworth Publishing Group, Belmont, California, ISBN-13:9780534097264,.

Bryman, A., 2004. Social Research Methods. 2nd Edn., Oxford University Press Inc., New York.

Bushko, R.G., 2009. Strategy for the Future of Health. IOS Press, Amsterdam, Netherlands, ISBN:9781607500506, Pages: 411.

Charmaz, K., 2006. Constructing Grounded Theory: A Practical Guide Through Qualitative Analysis. Sage Publications, Los Angeles.

Clark, F. and D.L. Illman, 2003. Content analysis of New York Times coverage of space issues for the year 2000. Sci. Commun., 25: 14-38.

Creswell, J.W., 2013. Qualitative Inquiry and Research Design: Choosing among five Approaches. 3rd Edn., Sage Publication, Thousand Oaks, California,

De Wever, B., T. Schellens, M. Valcke and H. van Keer, 2006. Content analysis schemes to analyze transcripts of online asynchronous discussion groups: A review. Comput. Educ., 46: 6-28.

Denzin, N.K. and Y.S. Lincoln, 2012. Introduction: The Discipline and Practices of Qualitative Resea In: Collecting and Interpreting Qualitative Materials, Denzin, N.K. and Y.S. Lincoln (Eds.)., SAGE Publications, Thousand Oaks, California, USA., ISBN:9781483307305, pp: 1-54.

Ellingson, L.L., 2012. Analysis and Representation Across the Continuum. In: Collecting and Interpreting Qualitative Materials, Denzin, N.K. and Y.S. Lincoln (Eds.)., SAGE Publications, Thousand Oaks, California, USA., ISBN:9781483307305, pp: 413-445.

Franzosi, R., 2008. Content analysis: Objective, systematic and quantitative description of content. Content Anal., 1: 21-50.

Hsieh, H.F. and S.E. Shannon, 2005. Three approaches to qualitative content analysis. Qual. Health Res., 15: 1277-1288.

Kohlbacher, F., 2006. The use of qualitative content analysis in case study research. Forum Qual. Soc. Res., 7: 1-30.

Kolbe, R.H. and M.S. Burnett, 1991. Content-analysis research: An examination of applications with directives for improving research reliability and objectivity. J. Consum. Res., 18: 243-250.

Kreuger, L. and W.L. Neuman, 2006. Social Work Research Methods: Qualitative and Quantitative Approaches, With Research Navigator. Pearson/Allyn and Bacon, Boston, Massachusetts, USA, ISBN:9780205470112, Pages: 624.

Lacy, S., B.R. Watson, D. Riffe and J. Lovejoy, 2015. Issues and best practices in content analysis. Journalism Mass Commun. Q., 92: 791-811.
Leah, R., B. Vande and L.A. Wermer, 1991. Television Criticism: Approaches and Applications. Longman, New York, USA., ISBN:9780801305801, Pages: 490.

Lincoln, Y.S. and E.G. Guba, 1985. Naturalistic Inquiry. Sage Publication, Beverly Hills, CA., USA., ISBN-13: 978-0803924314, Pages: 416.

Lombard, M., J. Snyder Duch and C.C. Bracken, 2002. Content analysis in mass communication: Assessment and reporting of intercoder reliability. Hum. Commun. Res., 28: 587-604.

Marshall, C. and G.B. Rossman, 1999. The what of the study: Building the conceptual framework. Designing Qual. Res., 3: 21-54.

McKee, A., 2001. A beginner's guide to textual analysis. Metro Mag., 127: 138-149.

Miles, M.B., and A.M. Huberman, 1994. Qualitative Data Analysis: An Expanded Sourcebook. SAGE, Thousand Oaks, California, ISBN:9780803955400, Pages: 338.

Millen, J.K. and T.F. Lunt, 1992. Security for object-oriented database systems. Proceedings of the 1992 IEEE International Symposium on Computer Society Research in Security and Privacy, May 4-6, 1992, IEEE, Oakland, California, USA., pp: $260-272$.

Minichiello, V., R. Aroni, E. Timewell and L. Alexander, 1990. In-Depth Interviewing: Researching People. Longman Cheshire, Hong Kong, ISBN-13: 9780582712720 , Pages: 357.

Moerman, M., 1992. Life after CA: An Ethnographer's Autobiography. In: Text in Context: Contributions to Ethnomethodology, Watson, G. and R.M. Seiler (Eds.)., SAGE, Newbury, Park, California, pp: 20-34.

Moy, P. and J. Murphy, 2016. Problems and prospects in survey research. J. Mass Commun. Q., 93: 16-37.

Neuendorf, K.A., 1989. The Content Analysis Guidebook. SAGE, Thousand, Oaks, California,.

Pasadeos, Y., B. Huhman, T. Standley and G. Wilson, 1995. Applications of content analysis in news research: A critical examination. Proceedings of the Annual Conference on Association for Education in Journalism and Mass Communication, May 26, 1995, Washington, USA., pp: 135-148.

Potter, W.J. and D. Levine Donnerstein, 1999. Rethinking validity and reliability in content analysis. J. Appl. Commun. Res., 27: 258-284.

Schilling, J., 2006. On the pragmatics of qualitative assessment. Eur. J. Psychol. Assess., 22: 28-37.

Sesay, M., 2011. Developmental students levels of engagement and student success in two-year institutions: A study of a suburban community college system in Texas. Ph.D Thesis, The University of Texas at Austin, Austin, Texas. 
Seward, N., D. Osrin, L. Li, A. Costello and A.M. Pulkki-Brannstrom et al., 2012. Association between clean delivery kit use, clean delivery practices and neonatal survival: Pooled anlalysis of data from three sites in South Asia. PLoS Med., 9: 1-11.

Van Dijk, T.A., 1985. Handbook of Discourse Analysis: Discourse Analysis in Society. Academic Press, Cambridge, Massachusetts, USA., ISBN:9780127120041, Pages: 228.
Weber, M.G., 1990. Forest soil respiration after cutting and burning in immature aspen ecosystems. For. Ecol. Manage., 31: 1-14.

Welsch, R.E., 1992. Data analysis, communication and control. Working Paper No. 64, National Bureau of Economic Research, Cambridge, Massachusetts, USA. https://www.nber.org/papers/w0064

Wimmer, R.D. and J.R. Dominick, 1994. Mass Media Research: An Introduction. 4th Edn., Wadsworth Publishing Group, Belmont, California, 